\title{
IRF2 contributes to myocardial infarction via regulation of GSDMD induced pyroptosis
}

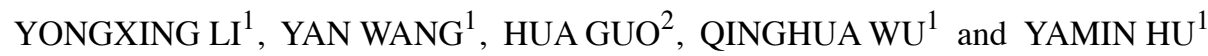 \\ ${ }^{1}$ Department of Cardiovascular Medicine, Cangzhou Central Hospital, Cangzhou, Hebei 061001; \\ ${ }^{2}$ Department of Cardiovascular Medicine, Hebei Province Cangzhou Hospital of \\ Integrated Traditional and Western, Cangzhou, Hebei 061001, P.R. China
}

Received August 14, 2021; Accepted October 28, 2021

DOI: $10.3892 / \mathrm{mmr} .2021 .12556$

\begin{abstract}
Interferon regulatory factor (IRF) 2 is a transcription factor belonging to the IRF family, which is essential for gasdermin D (GSDMD)-induced pyroptosis. Decreasing myocardial cell pyroptosis confers protection against heart damage and cardiac dysfunction caused by myocardial infarction (MI). The aim of the present study was to investigate the involvement of IRF2 in MI and the underlying mechanism of IRF2 in pyroptosis. To mimic MI, ligation of the left anterior descending coronary artery was performed to establish an in vivo mouse model and rat cardiomyocytes H9c2 cells were cultured under hypoxic conditions to establish an in vitro model. Transthoracic echocardiography was used to assess cardiac function. Hematoxylin and eosin staining was used to observe histopathological changes in the myocardial tissue. Immunohistochemistry and western blotting were performed to detect IRF2 expression levels. TUNEL staining and flow cytometry were used to detect apoptosis in myocardial tissue and cells. Chromatin immunoprecipitation and dual luciferase reporter assay were used to verify the effect of IRF2 on GSDMD transcription. IRF2 was upregulated in MI mice. MI induced pyroptosis, as evidenced by increased GSDMD, N-terminal GSDMD (GSDMD-N), and cleaved (c-) caspase-1 levels. MI increased IL-1 $\beta$ and IL-18 levels. These alterations were alleviated by IRF2 silencing. Furthermore, in hypoxia-treated $\mathrm{H} 9 \mathrm{c} 2$ cells, IRF2 silencing significantly decreased the elevated levels of IL-1 $\beta$ and IL-18 and pyroptosis-associated proteins, including GSDMD, GSDMD-N and c-caspase1. Moreover, in hypoxia-treated H9c2 cells, IRF2
\end{abstract}

Correspondence to: Dr Yamin Hu, Department of Cardiovascular Medicine, Cangzhou Central Hospital, 16 Xinhua West Road, Canal Zone, Cangzhou, Hebei 061001, P.R. China

E-mail: yaminhym@163.com

Abbreviations: IRF, interferon regulatory factor; MI, myocardial infarction; GSDMD, gasdermin D; EF, ejection fraction; FS, fractional shortening; c-, cleaved; ChIP, chromatin immunoprecipitation

Key words: IRF2, MI, GSDMD, pyroptosis, hypoxia directly bound to the GSDMD promoter to drive GSDMD transcription and promote pyroptosis and IRF2 expression may be regulated via the hypoxia inducible factor 1 signaling pathway. In conclusion, the present results demonstrated that IRF2 is a key regulator of MI by mediating pyroptosis, which triggers GSDMD activation.

\section{Introduction}

Myocardial infarction (MI) is a key manifestation of cardiovascular disease (1). According to the Centers for Medicare \& Medicaid Services Hospital Inpatient Quality Reporting Program data on 2,363 hospitals in 2018, the average mortality of acute MI was $13.6 \%$ (2). MI is the injury and necrosis of myocardial cells caused by a sudden decrease in coronary blood flow, continuous myocardial ischemia and hypoxia $(3,4)$. In clinical practice, the primary application of percutaneous coronary intervention is to promptly restore coronary blood transport and save the dying myocardium $(5,6)$. Left ventricular remodeling following MI can have serious consequences, such as heart failure and rupture, and is the primary cause of death in patients with MI (1). Therefore, delaying or preventing left ventricular remodeling is an important step in preventing heart failure following MI. However, the detailed mechanisms underlying MI remain unclear. Therefore, it is important to investigate the mechanisms of MI injury, which may help to develop novel therapies for myocardial injury.

Programmed cell death includes necroptosis, parthanatos, oxytosis, ferroptosis, extracellular trap (ET)osis, neutrophil ETosis and pyroptosis (7). Pyroptosis leads to myocardial perforation and rupture of the cell membrane and releases pro-inflammatory factors such as interleukin-1 $\beta$ (IL-1 $1 \beta$ ) and IL-18, initiated by caspase-1, causing a local inflammatory response (8). Previous studies have found that pyroptosis serves an important role in cardiovascular disease progression (including atherosclerosis, MI, ischemia-reperfusion, diabetic cardiomyopathy and heart failure) (9-11).

A previous study indicated that the inflammatory body-caspase axis serves an important role in inflammation-induced cell death following ischemia-reperfusion (10). The inflammasome is a complex that can transform pro-caspase-1 into activated caspase-1. Activated caspase-1 can convert pro-IL-1 $\beta$ and pro-IL-18 into mature IL-1 $\beta$ and 
IL-18, cleave gasdermin D (GSDMD) into the GSDMD-N terminal with a pore-forming effect and induce pyroptosis (12). GSDMD, a member of the GSDM family, is a common substrate for caspase-1,-4, -5 and -11 and mediates pyroptosis by producing an $\mathrm{N}$-terminal fragment $(13,14)$. Active caspase-1, -4 -11 cleave GSDMD in response to canonical and non-canonical inflammasome activation (15). Moreover, GSDMD inhibition improves cardiac function and decreases infarct size by attenuating cardiomyocyte pyroptosis $(16,17)$. The role of pyroptosis and the mechanism of its occurrence and development in the MI process remain to be elucidated, which may provide novel directions and strategies for the clinical treatment of cardiovascular disease.

Interferon regulators (IRFs) are multifunctional transcription factors that serve an important regulatory role in the interferon signaling pathway, which is involved in host immune response, cell differentiation and immune regulation (18). IRF2 is a member of the IRF family of transcription factors (19), which can transcriptionally induce direct target genes, including toll-like receptor 3, Bcl-11a and GSDMD (20-22). Kayagaki et al (22) found that IRF2 transcriptionally induces GSDMD expression in pyroptosis and directly drives GSDMD mRNA expression. GSDMD expression is significantly decreased in IRF2-deficient macrophages, endothelial cells and multiple types of tissue, corresponding to decreased IL-1 secretion and inhibition of pyroptosis (22). However, its functional contribution to MI remains unclear and requires further clarification.

The present study investigated the effect of IRF2 in MI injury and clarified the potential mechanism of IRF2 involvement in MI via GSDMD-induced pyroptosis.

\section{Materials and methods}

Bioinformatics analysis. GeneMANIA (23), a web tool that can identify other proteins associated with a set of input genes, was used to generate protein-protein interaction (PPI) network images. The associations between co-expression, colocalization, predicted related genes, shared protein domains, genetic interactions and physical interactions were determined using GeneMANIA.

Animals and MI model in vivo. A total of 25 male C57BL/6 mice (aged 6-8 weeks; weight, 20-26 g) were provided by Ai Ling Fei (Nanjing, China). Mice were maintained at $23-25^{\circ} \mathrm{C}$ with $60-70 \%$ humidity, 12-h light/dark cycles and access to normal chow diet and water. The mice were divided into the following five groups ( $\mathrm{n}=5$ per group): i) Control (Con); ii) sham; iii) MI; iv) $\mathrm{MI}+$ sh-NC; and v) MI + sh-IRF2. Untreated mice were set as the con group. For shRNAs transfection in vivo, mice were injected with lentivirus-IRF2-short hairpin (sh)RNA (sh-IRF2 forward, GGTCCTGACTTCAACTATA; reverse, TATAGT TGAAGTCAGGACC) or lentivirus-scramble (forward, UUCUCCGAACGUGUCACGUTT; reverse, ACGUGACAC GUUCGGAGAATT) (both $1 \times 10^{9}$ viral particles/mouse, Shanghai GenePharma Co., Ltd.) via the tail vein. After 7 days, a mouse model of MI was generated by ligation of the left anterior descending coronary artery, as previously described (24). Briefly, mice were anesthetized with $45 \mathrm{mg} / \mathrm{kg}$ $1 \%$ pentobarbital sodium intraperitoneal injection and then underwent open-heart surgery. The MI model was established by threading a $7 / 0$ surgical thread $2 \mathrm{~mm}$ under the left auricle and ligating the anterior descending branch of the left coronary artery for $24 \mathrm{~h}$. In the sham group, a parallel operation was performed, however, the thread was threaded without ligation. After operation, 40,000 units of penicillin (Thermo Fisher Scientific, Inc.) were injected intraperitoneally daily for 3 consecutive days.

Cell culture and MI model in vitro. $\mathrm{H} 9 \mathrm{c} 2$ cells were purchased from EK Biosciences GmbH (Shanghai, China) and cultured in Dulbecco's Modified Eagle Medium containing 10\% foetal bovine serum (both Thermo Fisher Scientific, Inc.) at $37^{\circ} \mathrm{C}$ with $5 \% \mathrm{CO}_{2}$. To establish an MI injury model in vitro, $\mathrm{H} 9 \mathrm{c} 2$ cells were exposed to a hypoxia incubator $\left(1 \%\right.$ oxygen, $5 \% \mathrm{CO}_{2}$ and $94 \% \mathrm{~N}_{2}$ ) for $6,12,24$ and $48 \mathrm{~h}$. Untreated cells were set as Con group.

Histopathological examination and immunohistochemical (IHC) staining. At $24 \mathrm{~h}$ after the surgery, the were mice were euthanized by intraperitoneal injection of $50 \mathrm{mg} / \mathrm{kg}$, followed by dislocation of the cervical spine. The heart tissue samples were fixed in $4 \%$ paraformaldehyde at room temperature for $24 \mathrm{~h}$, embedded in paraffin and sectioned into $5 \mu \mathrm{m}$ slices. The slices were stained with hematoxylin-eosin $(\mathrm{H} \& \mathrm{E})$ for $7 \mathrm{~min}$ at room temperature and photographed using a light microscope (x400 magnification; Nikon Eclipse TE2000-U; Nikon Corporation). For IHC staining of IRF2, the tissue slices were deparaffinized in xylene, dehydrated with graded ethanol solutions and rehydrated in PBS. The slices then were incubated with $3 \% \mathrm{H}_{2} \mathrm{O}_{2}$ at $37^{\circ} \mathrm{C}$ for 10 min to block endogenous peroxidase activity. For antigen retrieval, the slices were treated with PBS ( $\mathrm{pH}$ 9) for $15 \mathrm{~min}$ at $95^{\circ} \mathrm{C}$ in a microwave oven and then cooled in running water. Subsequently, slices were incubated with normal goat serum sealant (Shanghai Haoran Biotechnology Co., Ltd.) for $20 \mathrm{~min}$ at room temperature. Slices were then incubated with anti-IRF2 antibody (cat. no. PA5-83159; 1:200; Sigma-Aldrich; Merck KGaA) in a humidified chamber at $4^{\circ} \mathrm{C}$ overnight and were visualized with $\mathrm{DAB}$ at room temperature for $2 \mathrm{~min}$ and counterstained with hematoxylin at room temperature for $5 \mathrm{~min}$.

Echocardiographic measurement. Transthoracic echocardiography was performed $24 \mathrm{~h}$ after surgery in mice post-MI using an echocardiography system equipped with a $12-\mathrm{MHz}$ phased-array transducer. The left ventricular ejection fraction (EF) and fractional shortening (FS) were measured using M-mode tracing. All experiments were performed by professional technicians who were blinded to the experimental groups.

Western blotting. Total protein samples from H9c2 cells and heart tissue were extracted by using RIPA lysis buffer containing $1 \mathrm{mM}$ phenylmethylsulfonyl fluoride (Beyotime Institute of Biotechnology). The total protein concentrations were determined using a bicinchoninic acid Protein Assay kit (Beyotime Institute of Biotechnology). Proteins (30 $\mu \mathrm{g}$ per lane) were separated using 10\% SDS-PAGE (Beyotime Institute of Biotechnology) and then transferred to PVDF membranes. The membranes were blocked in 5\% skimmed 
milk for $1 \mathrm{~h}$ and then incubated overnight at $4^{\circ} \mathrm{C}$ with primary antibodies. The blot was probed using the following primary antibodies: IRF2 (cat. no. A303-380A-T; 1:1,000; Thermo Fisher Scientific, Inc.), cleaved caspase-1 (c-caspase-1; cat. no. PA5-77886; 1:1,000; Thermo Fisher Scientific, Inc.), GSDMD (cat.no.PA5-30823; 1:1,000; Thermo Fisher Scientific, Inc.), GSDMD-N (cat. no. ab215203; 1:1,000; Abcam), IL-1 $\beta$ (cat. no. P420B; 1:1,000; Thermo Fisher Scientific, Inc.), IL-18 (cat. no. PA5-79477; 1:1,000; Thermo Fisher Scientific, Inc.), and GAPDH (cat. no. ab8245; 1:5,000; Abcam), followed by incubation with goat anti-rabbit IgG secondary antibody (cat. no. G-21234; 1:10,000; Thermo Fisher Scientific, Inc.) at room temperature for $1 \mathrm{~h}$. Protein bands were visualized by enhanced chemiluminescence Pierce ${ }^{\mathrm{TM}}$ ECL Western Blotting Substrate (cat. no. 32209; Thermo Fisher Scientific, Inc.). The densitometry analysis was performed using ImageJ software (version 1.8.0; National Institutes of Health).

TUNEL staining. Myocardial tissue apoptosis was measured using a In Situ Cell Death Detection Kit (Sigma Aldrich; Merck KGaA) according to the manufacturer's instructions. For the TUNEL staining, the heart tissue samples were fixed in $4 \%$ paraformaldehyde at room temperature for $24 \mathrm{~h}$, embedded in paraffin and sectioned into 5- $\mu \mathrm{m}$ slices. The slices were deparaffinized in xylene, dehydrated with graded ethanol solutions and rehydrated in PBS, and then treated with $25 \mu \mathrm{g} / \mathrm{ml}$ proteinase $\mathrm{K}$ for $8 \mathrm{~min}$ at room temperature. Then, the slices were stained with the TUNEL reaction mixture containing TdT and TMR-dUTP for $1 \mathrm{~h}$ at $37^{\circ} \mathrm{C}$ in the dark. After applying stop solution for $10 \mathrm{~min}$ at room temperature, streptavidin-HRP working solution $(50 \mu \mathrm{l})$ was added at room temperature for $30 \mathrm{~min}$, followed by incubation with DAB $(50 \mu \mathrm{l})$ solution at room temperature for $10 \mathrm{~min}$. The color was then developed using 3,3-diaminobenzidine (Sigma-Aldrich; Merck KGaA). A total of five fields of view were randomly selected to count the TUNEL-positive cells under a light microscope (x400 magnification) and the proportion of TUNEL-positive cells was calculated.

Determination of cellular apoptosis. H9c2 cells were cultured at room temperature in 6-well plates at a density of $1 \times 10^{5}$ cells/well to evaluate pyroptosis. After hypoxia treatment, the treated $\mathrm{H} 9 \mathrm{c} 2$ cells were harvested and incubated with Annexin V-fluorescein isothiocyanate and propidium iodide for $15 \mathrm{~min}$ at $4^{\circ} \mathrm{C}$. The samples were analyzed using a flow cytometer (BD Accuri C6 Plus; BD Biosciences) with FlowJo software (v10.6.2; FlowJo, LLC) according to the manufacturer's instructions.

Chromatin immunoprecipitation (ChIP) assay. The ChIP assay was performed with the ChIP Assay kit (cat. no. P2078; Beyotime Institute of Biotechnology) according to the manufacturer's protocol. Briefly, $\mathrm{H} 9 \mathrm{c} 2$ cells $\left(1 \times 10^{5}\right)$ were incubated with $1 \%$ formaldehyde for $10 \mathrm{~min}$ at $25^{\circ} \mathrm{C}$ to crosslink the protein and DNA. Glycine (Beijing Solarbio Technology Co., Ltd.) was then used to terminate the crosslinking. $\mathrm{H} 9 \mathrm{c} 2$ cells were centrifugated at $1,000 \times \mathrm{g}$ for $2 \mathrm{~min}$ at $4^{\circ} \mathrm{C}$, resuspended in the lysis buffer $(0.2 \mathrm{ml})$ at at $4^{\circ} \mathrm{C}$ for $10 \mathrm{~min}$. The $\mathrm{Ch}$ fragments were obtained by shearing crosslinked complex DNA with ultrasound, followed by IP with the antibody directed against anti-IRF2 (cat. no. ab245658; 1:100; Abcam) overnight at $4^{\circ} \mathrm{C}$, taking IgG as a negative control. $\mathrm{Ch}$ fragments were detected by PCR.

RNA extraction and reverse transcription-quantitative PCR $(R T-q P C R)$. Total RNA was isolated from H9c2 cells using RNAsimple Total RNA Kit (cat. no. DP419; Tiangen Biotech Co., Ltd.) and RNA was reverse transcribed into cDNA using a PrimeScript ${ }^{\circledR}$ RT reagent kit (Takara Bio, Inc.) according to the manufacturer's protocol. Subsequently, qPCR was performed using the SYBR Premix Ex Taq ${ }^{\mathrm{TM}}$ II kit (Thermo Fisher Scientific, Inc.) and an ABI Prism 7700 sequence detector (Applied Biosystems; Thermo Fisher Scientific, Inc.). The sequences of the PCR primers used were as follows: GSDMD forward, 5'-TCTGCCCTCAACACTTCTGG-3' and reverse, 5'-TGCAGCCACAAATAACTCAGC-3'; and GAPDH forward, 5'-GAAGGTCGGAGTCAACGGATT-3' and reverse, 5'-TTCCCGTTCTCAGCCATGT-3'. The following thermocycling conditions were used: $90^{\circ} \mathrm{C}$ for $5 \mathrm{~min}$; then $90^{\circ} \mathrm{C}$ for $15 \mathrm{sec}$ and $60^{\circ} \mathrm{C}$ for $30 \mathrm{sec}$ for 45 cycles. Expression levels were quantified using the $2^{-\Delta \Delta \mathrm{Cq}}$ method (25) and normalized to the internal reference gene GAPDH.

Dual-luciferase reporter assay. The recombinant luciferase reporter plasmids containing the GSDMD promoter (pEZX-PG04-GSDMD), pcDNA3.1 vector (pc-NC, $100 \mathrm{nM}$ ), IRF2 overexpression vector (IRF2, $100 \mathrm{nM}$ ), IRF2 short hairpin (sh)RNA (sh-IRF2 forward, GGTCCTGACTTCAACTATA; reverse, TATAGTTGAAGTCAGGACC; $100 \mathrm{nM}$ ) or negative control (sh-NC forward, UUCUCCGAACGUGUCACG UTT; reverse, ACGUGACACGUUCGGAGAATT; $100 \mathrm{nM}$ ) were co-transfected into $\mathrm{H} 9 \mathrm{c} 2$ cells by Lipofectamine ${ }^{\mathrm{TM}}$ 3000 transfection reagent (cat. no. L3000001; Thermo Fisher Scientific, Inc.) for $48 \mathrm{~h}$ at $37^{\circ} \mathrm{C}$. Following $48 \mathrm{~h}$ transfection, the luciferase activity was detected using a Dual-Luciferase Reporter Gene Assay Kit (cat. no. 11402ES60; Shanghai Qcbio Science \& Technologies Co., Ltd.) according to the manufacturer's instructions. The firefly luciferase activity was normalized to Renilla luciferase activity for each sample.

Statistical analysis. All data were analyzed using GraphPad Prism 9 (GraphPad Software, Inc.) and are expressed as the mean \pm SD. All experiments were repeated three times. One-way ANOVA followed by Tukey's multiple comparisons test was used for comparisons between groups. $\mathrm{P}<0.05$ was considered to indicate a statistically significant difference.

\section{Results}

IRF2 expression is associated with MI in mice. $\mathrm{H} \& \mathrm{E}$ and IHC staining for IRF2 showed severe MI in MI model mice. IHC analysis revealed a positive association between increased IRF2 expression and MI progression (Fig. 1A and B). Western blotting indicated that IRF2 expression was increased in MI samples compared with that in the con (Fig. 1C). Taken together, these results indicated that IRF2 expression was positively associated with MI progression.

IRF2 silencing attenuates MI in mice. The effect of IRF2 on MI was investigated. IRF2 shRNA was transmitted to mice using a lentivirus. IRF2 silencing alleviated histological heart 
A

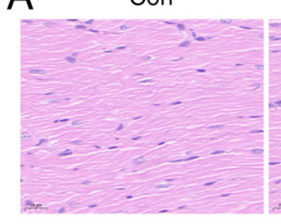

B

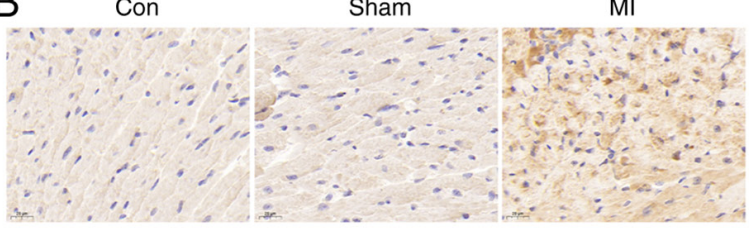

$\mathrm{MI}$

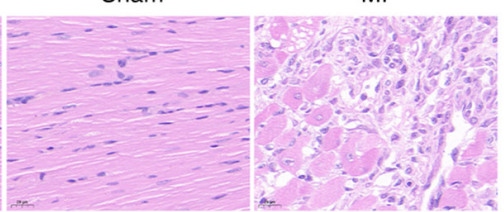

MI
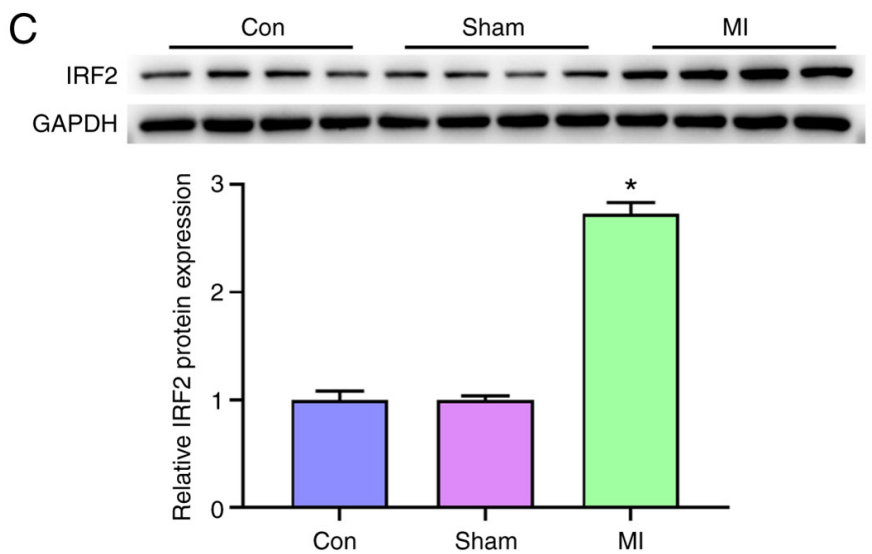

Figure 1. IRF2 expression is associated with MI in mice. (A) Representative hematoxylin and eosin staining in the left ventricle of mice. (B) IRF2 expression in heart tissue of MI mice. Scale bar, $20 \mu \mathrm{m}$. (C) Western blotting was used to detect the expression of IRF2 in heart tissue of MI mice. All data are expressed as the mean $\pm \mathrm{SD}(\mathrm{n}=3)$. ${ }^{*} \mathrm{P}<0.05$ vs. Sham. IRF2, interferon regulatory factor 2; MI, myocardial infarction; Con, control.

A

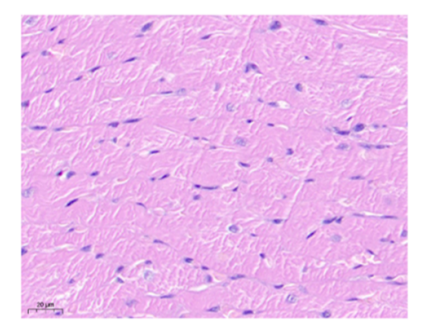

$\mathrm{B}$

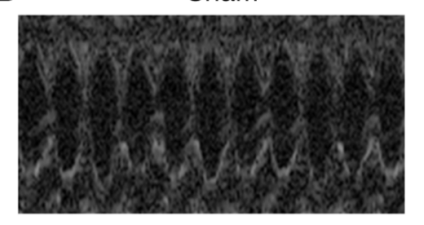

C

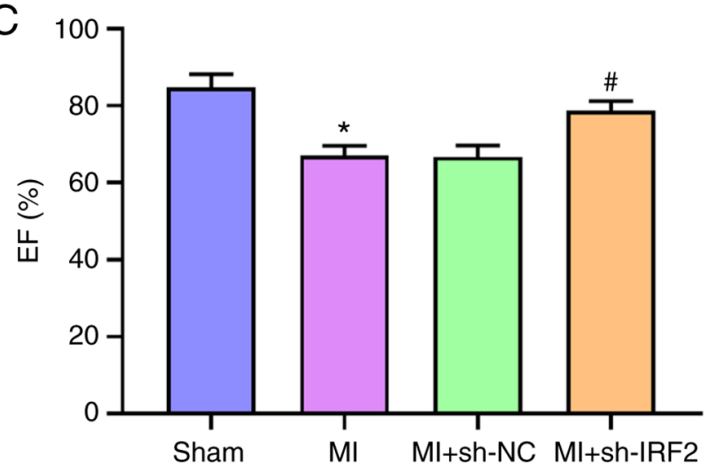

$\mathrm{Ml}+\mathrm{sh}-\mathrm{NC}$

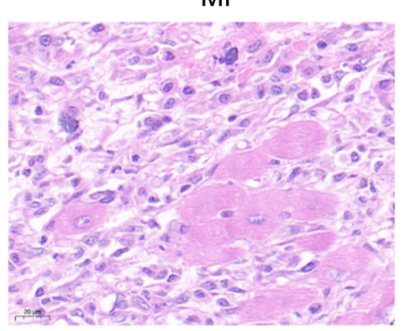

$\mathrm{MI}$
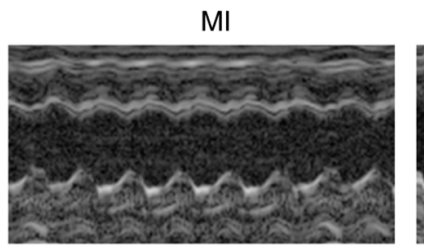

$\mathrm{Ml}+\mathrm{sh}-\mathrm{NC}$
$\mathrm{MI}+\mathrm{Sh}-\mathrm{IRF2}$
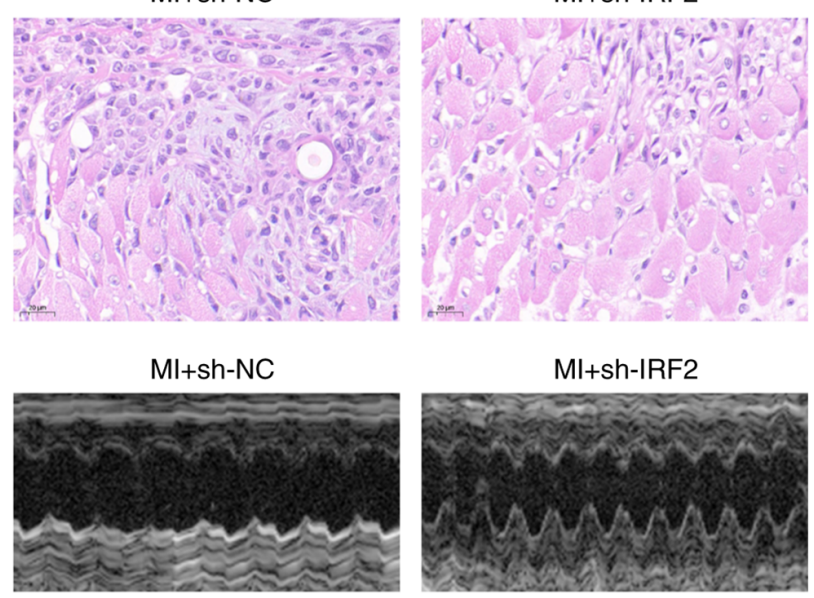

D

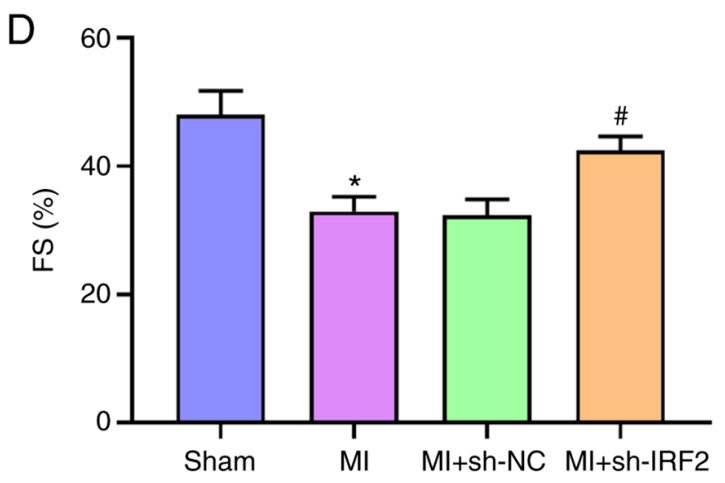

Figure 2. IRF2 silencing attenuates MI in mice. (A) Representative hematoxylin and eosin staining in the left ventricle of mice following IRF2 silencing. Scale bar, $20 \mu \mathrm{m}$. Arrows indicate alleviation of histological heart damage. (B) After silencing IRF2, cardiac function of mice was measured by transthoracic echocardiography to assess (C) EF and (D) FS. All data are expressed as the mean $\pm \mathrm{SD}(\mathrm{n}=3)$. ${ }^{*} \mathrm{P}<0.05$ vs. Sham; ${ }^{\prime} \mathrm{P}<0.05$ vs. MI + sh-NC. IRF2, interferon regulatory factor 2; MI, myocardial infarction; EF, ejection fraction; FS, fractional shortening; sh, short hairpin; NC, negative control.

damage in the $\mathrm{MI}+$ sh-IRF2 group compared with the MI group (Fig. 2A). To understand the effects of IRF2 silencing on MI mice, in vivo cardiac function was measured using transthoracic echocardiography (Fig. 2B). Compared with the sham group, MI significantly decreased EF and FS; this was improved in the MI + sh-IRF2 group (Fig. 2C and D). These data suggested that IRF2 silencing alleviated systolic dysfunction in MI mice.
IRF2 silencing attenuates GSDMD-induced pyroptosis in vivo. Pyroptosis, a new form of inflammatory programmed cell death, has been shown to aggravate MI following cardiomyocyte loss (16). To investigate the protective effect of IRF2 silencing on MI by decreasing pyroptosis, TUNEL staining was used to evaluate pyroptotic cell death in the MI model. Cardiac pyroptosis and the number of TUNEL-positive cells increased in the MI group and IRF2 silencing significantly 
A

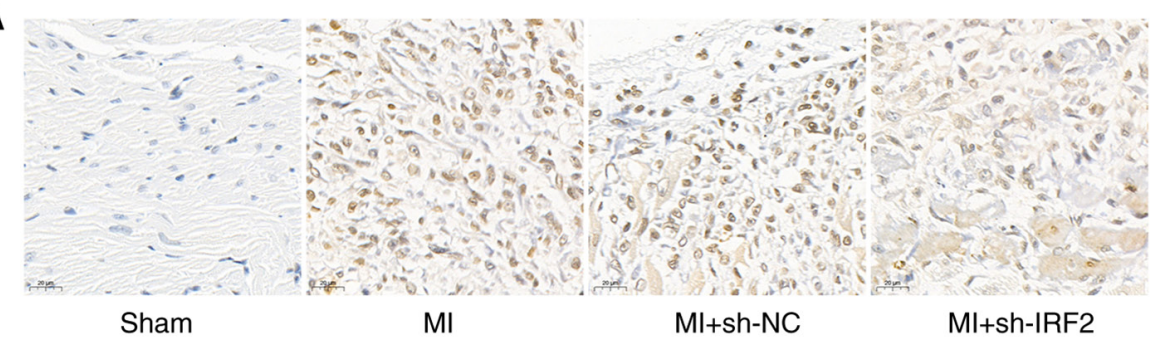

B

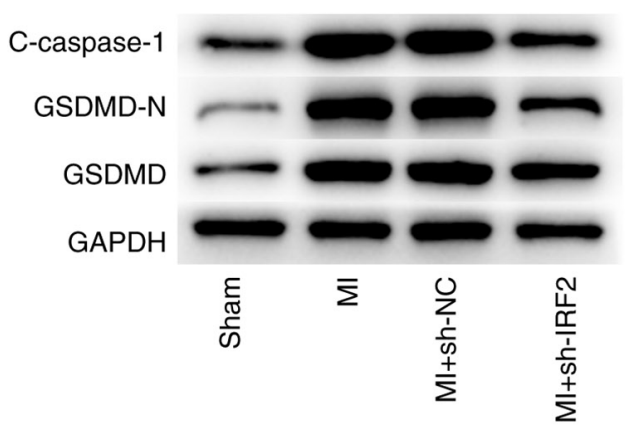

C

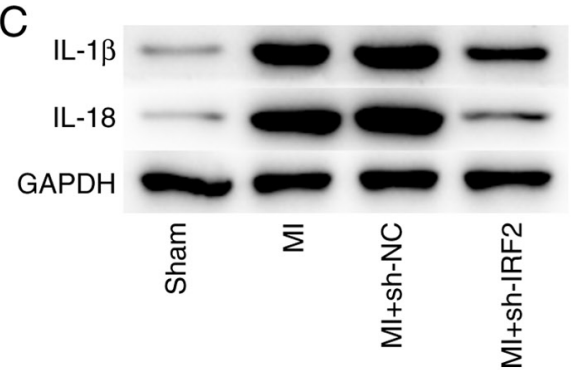

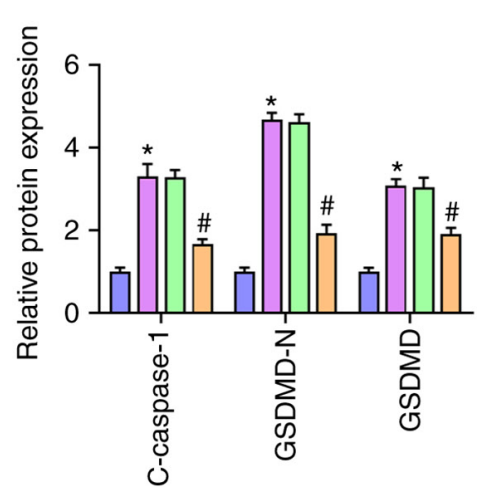

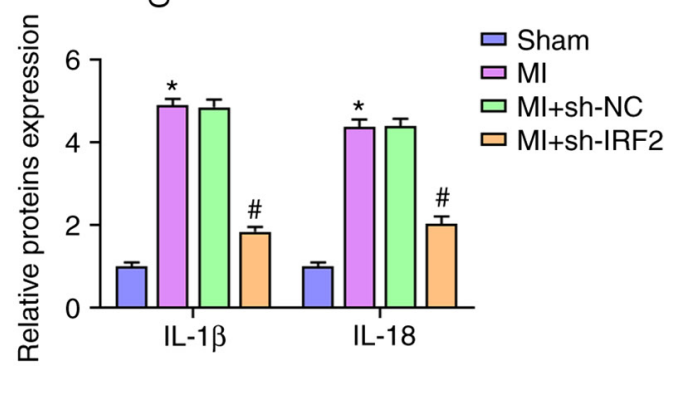

$\square \mathrm{Ml}+$ sh-IRF2

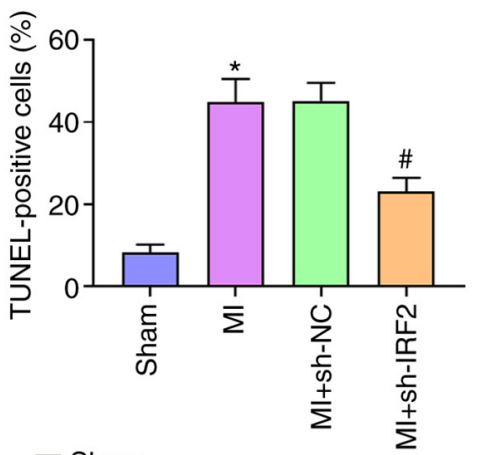

$\square \mathrm{MI}$

$\square \mathrm{Ml}+$ sh-NC

Figure 3. IRF2 silencing attenuates GSDMD-induced pyroptosis in vivo. (A) Myocardial apoptosis of mice was detected by TUNEL staining. Scale bar, $20 \mu \mathrm{m}$. (B) Effect of silencing IRF2 on expression of (B) c-caspase-1, GSDMD-N and GSDMD and (C) IL-1 $\beta$ and IL-18 in heart tissue of MI model mice. All data are expressed as the mean $\pm \mathrm{SD}(\mathrm{n}=3)$. ${ }^{*} \mathrm{P}<0.05$ vs. Sham; ${ }^{\mathrm{P}}<0.05$ vs. MI + sh-NC. IRF2, interferon regulatory factor 2 ; GSDMD, gasdermin $\mathrm{D}$; c-, cleaved; $\mathrm{MI}$, myocardial infarction; sh, short hairpin; NC, negative control.

inhibited cardiac pyroptosis (Fig. 3A). Western blot analysis revealed that $\mathrm{MI}$ resulted in the upregulation of c-caspase-1, IL-1 $\beta$, IL-18, GSDMD-N and GSDMD $24 \mathrm{~h}$ after MI; IRF2 silencing significantly inhibited expression of c-caspase-1, IL-1 $\beta$, IL-18, GSDMD-N and GSDMD protein levels induced by $\mathrm{MI}$ (Fig. 3B and C).

IRF2 silencing attenuates GSDMD-induced pyroptosis in vitro. One of the key events in MI is hypoxia, which also promotes pyroptosis $(26,27)$. To determine whether IRF2 silencing prevented pyroptosis in cardiomyocytes, H9c2 cells were cultured under hypoxic conditions for 6,12 , 24 and 48 h. Exposure of H9c2 cells to hypoxia (6-48 h) induced a time-dependent increase in IRF2 expression (Fig. 4A). Compared with the NC group, IRF2 expression was significantly decreased in the sh-IRF2 group and significantly increased in the IRF2 overexpression group (Fig. 4B). Hypoxia markedly elevated the rate of programmed cell death, and IRF2 silencing significantly decreases the rate of programmed cell death, and IRF2 overexpression aggravated the rate of programmed cell death compared with the hypoxia group (Fig. 4C). Western blot analysis showed that hypoxia resulted in the upregulation of c-caspase-1, IL-1 $\beta$, IL-18, GSDMD-N and GSDMD (Fig. 4D and E). Moreover, IRF2 silencing significantly inhibited the protein expression of c-caspase-1, IL-1 $\beta$, IL-18, GSDMD-N and GSDMD induced by hypoxia. These results suggested that IRF2 silencing attenuated GSDMD-induced pyroptosis in vitro.

IRF2 is key for the transcriptional activation of GSDMD. Co-expression of IRF2 and upstream genes recorded in GeneMANIA (28) (genemania.org/) showed that IRF2 may interact with HIF-1, indicating an association between HIF-1 and IRF2 (Fig. 5A). Exposure of H9c2 cells to hypoxia (6-48 h) induced a time-dependent increase in the expressions of IRF2 and HIF-1 (Fig. 5B). These data suggested that IRF2 expression may be regulated by the HIF-1 pathway in hypoxia-stimulated H9c2 cells. To verify whether IRF2 directly drives GSDMD in hypoxia-induced H9c2 cells, ChIP-PCR and dual-luciferase reporter assay were used to evaluate effect of IRF2 on GSDMD transcriptional activity. ChIP-PCR analysis of H9c2 cells using specific antibodies against IRF2 showed IRF2 was bound to the GSDMD promoter (Fig. 5C). Furthermore, dual-luciferase reporter assay showed that IRF2 overexpression increased GSDMD promoter activity and IRF2 silencing decreased GSDMD 
A

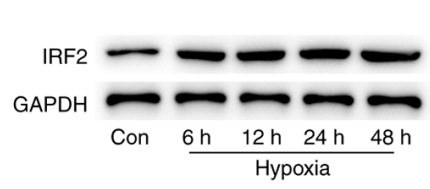

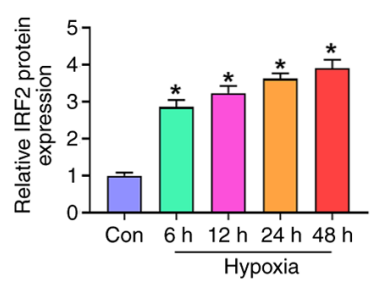

B

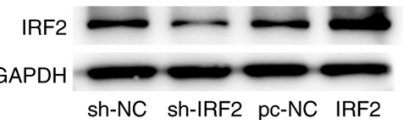

sh-NC sh-IRF2 pc-NC IRF2
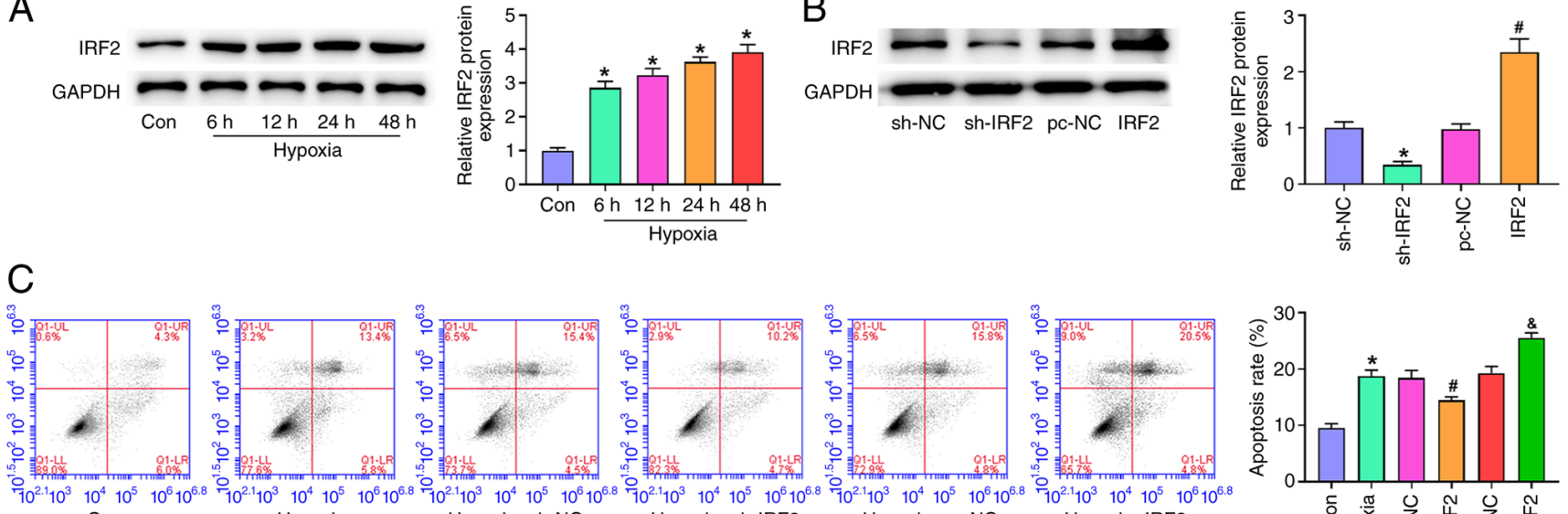

Con

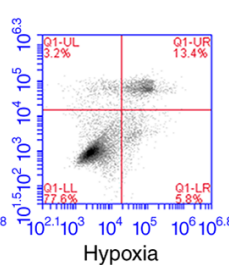

Hypoxia

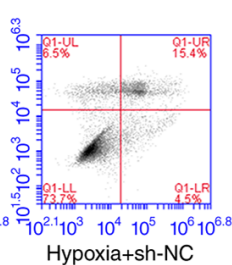
Hypoxia+sh-NC

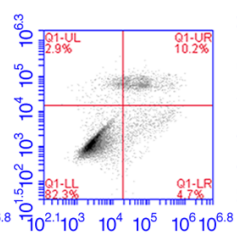
Hypoxia+sh-IRF2
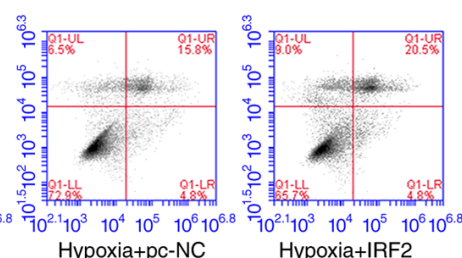

$\mathrm{E}$
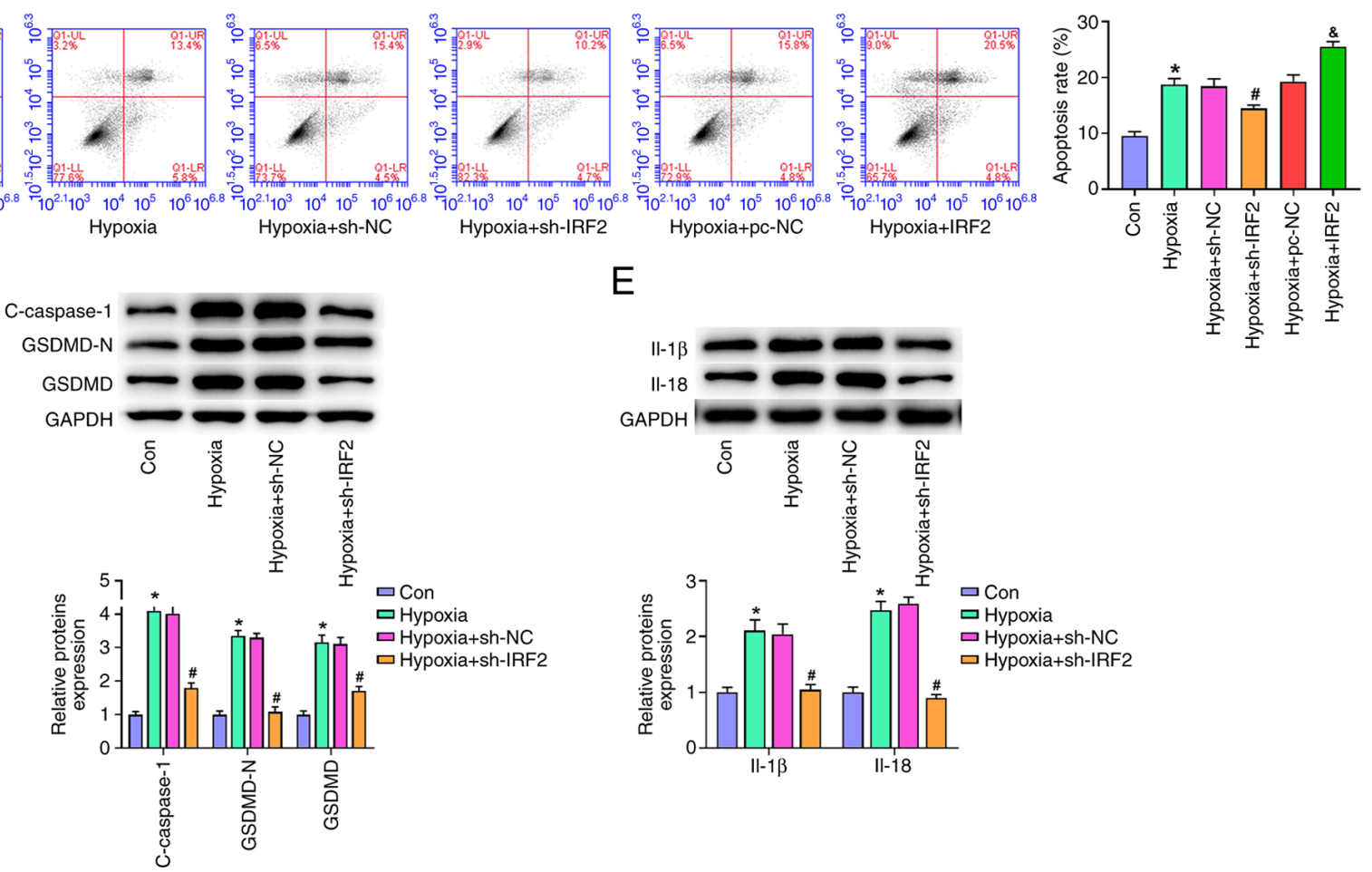

Figure 4. IRF2 silencing attenuates GSDMD-induced pyroptosis in vitro. (A) IRF-2 expression in H9c2 cells stimulated by hypoxia (6, 12,24 and 48 h) was detected by reverse transcription-quantitative PCR. ${ }^{*} \mathrm{P}<0.05$ vs. Con. (B) IRF2 expression was detected by western blotting. ${ }^{*} \mathrm{P}<0.05$ vs. sh-NC; ${ }^{\prime} \mathrm{P}<0.05$ vs. pc-NC. (C) Following transfection of sh-IRF2 and IRF2, apoptosis of H9c2 cells was detected by flow cytometry under hypoxic conditions. ${ }^{\&} \mathrm{P}<0.05 \mathrm{vs}$. Hypoxia + pc-NC, ${ }^{*} \mathrm{P}<0.05$ vs. Con; ${ }^{*} \mathrm{P}<0.05$ vs. Hypoxia+sh-NC. Following transfection of sh-IRF2 and IRF2, the expression of (D) c-caspase-1, GSDMD-N, GSDMD, (E) IL-1 3 , and IL-18 was detected by western blotting under hypoxic conditions. ${ }^{*} \mathrm{P}<0.05$ vs. Con; ${ }^{*} \mathrm{P}<0.05$ vs. Hypoxia + sh-NC. All data are expressed as the mean \pm SD $(\mathrm{n}=3)$. IRF2, interferon regulatory factor 2; GSDMD, gasdermin D; c-, cleaved; MI, myocardial infarction; sh, short hairpin; NC, negative control; Con, control.

promoter activity (Fig. 5D). These findings demonstrated that IRF2 bound directly to the GSDMD promoter. Western blot analysis showed that treatment of cells with hypoxia and sh-IRF2 inhibited c-caspase-1, GSDMD-N, and GSDMD protein expression in $\mathrm{H} 9 \mathrm{c} 2$ cells (Fig. 5E). Moreover, GSDMD overexpression increased the expression levels of c-caspase-1, GSDMD-N and GSDMD. These data suggested that IRF2 silencing alleviated MI and hypoxia-induced cardiomyocyte pyroptosis via transcriptional activation of GSDMD.

\section{Discussion}

The present study addressed the key role of IRF2 in MI. First, IRF2 expression was significantly increased in an MI mouse model and hypoxia-treated $\mathrm{H} 9 \mathrm{c} 2$ cells, and the level of IRF2 expression was positively associated with duration of hypoxia in H9c2 cells. Second, IRF2 silencing alleviated MI and hypoxia-induced cardiomyocyte pyroptosis in vivo and in vitro. Third, IRF2 was key for the transcriptional activation of GSDMD in cardiomyocytes. Therefore, IRF2 is a novel therapeutic target for treating MI.
Despite the use of advanced therapeutic interventions (29,30), MI remains the leading cause of death from cardiovascular disease (31). Pyroptosis is a newly identified form of programmed cell death that is mediated by inflammatory caspase-1, accompanied by release of a large number of pro-inflammatory factors and induces a cascade of amplified inflammatory responses (32); it is associated with numerous diseases, including MI and myocardial cell loss following ischemia-reperfusion $(33,34)$. IRF2 is a member of the IRF family and combines with interferon gene promoters to serve a key role in immune response and promote cell proliferation (35). Previous studies found that IRF2 is involved in various cancer mechanisms, including liver, lung and gastric cancer (36-38). To the best of our knowledge, however, there are few reports of its involvement in cardiovascular disease, particularly in MI. Recently, Kayagaki et al (22) found that IRF2 transcription induces GSDMD expression to cause pyroptosis. Therefore, it was hypothesized that IRF2 may erve a role in MI by regulating GSDMD-induced pyroptosis. In the present study, IRF2 expression was positively associated with MI progression and IRF2 silencing attenuated MI in mice. 
A
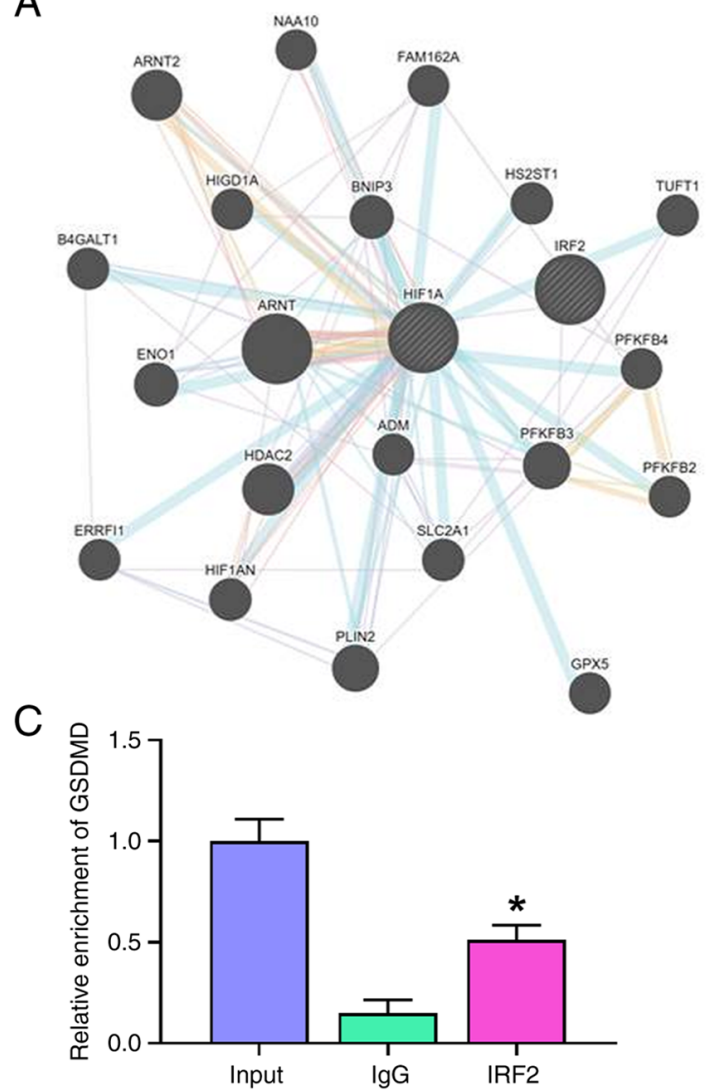

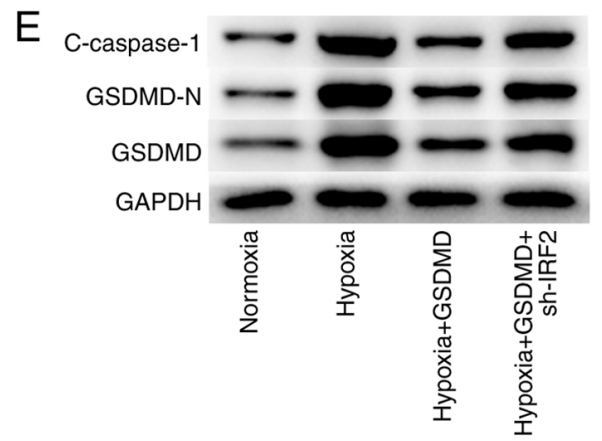

B
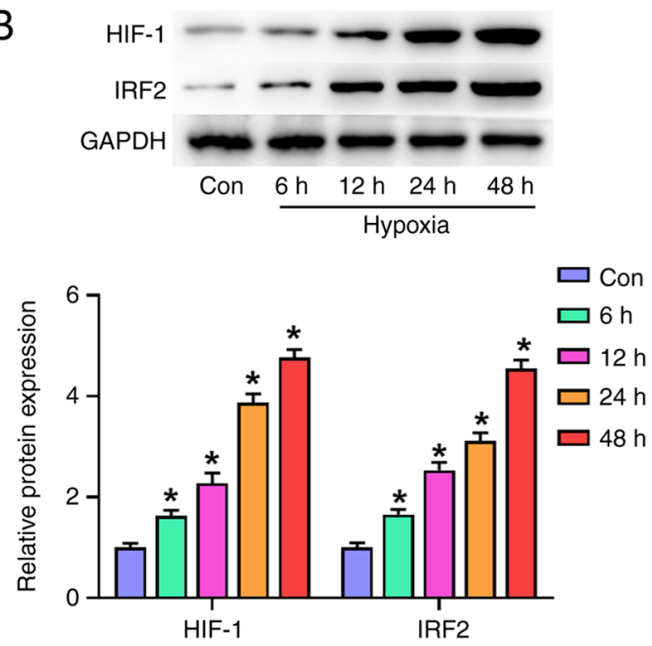

D
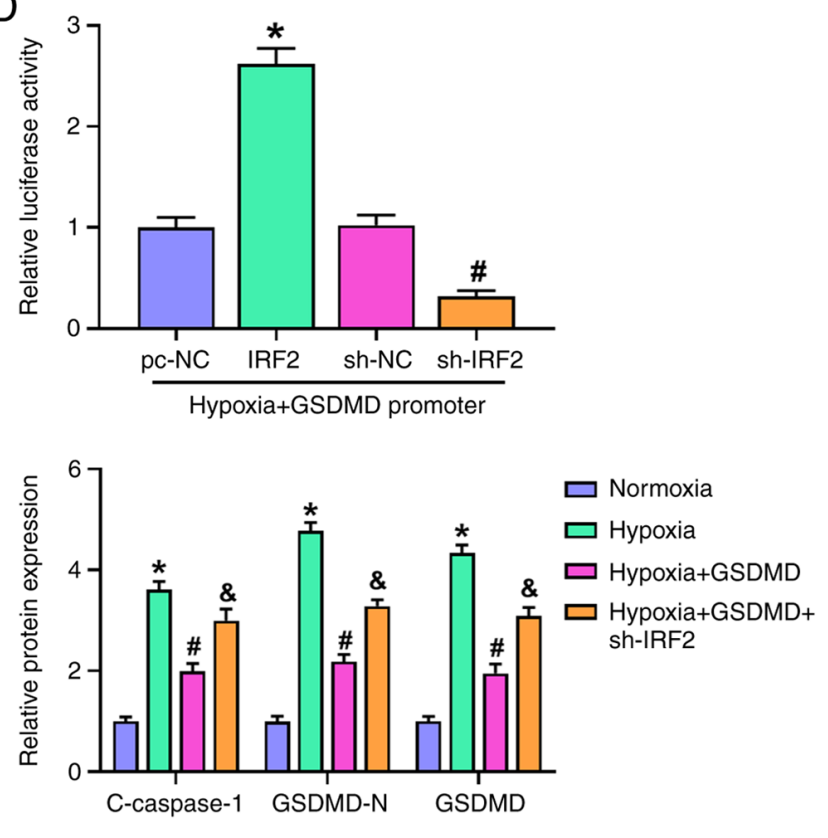

Figure 5. IRF2 is key for the transcriptional activation of GSDMD. (A) Co-expression of IRF2 and upstream genes was detected in GeneMANIA database. (B) HIF-1 and IRF-2 expression in H9c2 cells were stimulated following hypoxia (6, 12, 24 and 48 h). * P<0.05 vs. Con. (C) Chromatin immunoprecipitation-PCR was used to evaluate the transcriptional activity of IRF2 on GSDMD. ${ }^{*} \mathrm{P}<0.05$ vs. IgG. (D) Dual-luciferase reporter assay was used to evaluate the effect of IRF2 on GSDMD transcriptional activity. ${ }^{*} \mathrm{P}<0.05$ vs. pc-NC; ${ }^{\text {P }}<0.05$ vs. sh-NC. (E) Following transfection of sh-IRF2 and GSDMD, the expression of c-caspase-1, GSDMD-N and GSDMD was detected by western blotting under hypoxic conditions. $\mathrm{P}<0.05$ vs. Normoxia; ${ }^{\sharp} \mathrm{P}<0.05$ vs. Hypoxia; ${ }^{\&} \mathrm{P}<0.05$ vs. Hypoxia + GSDMD. All data are expressed as the mean \pm SD $(n=3)$. IRF2, interferon regulatory factor 2; GSDMD, gasdermin D; HIF-1, hypoxia inducible factor-1; Con, control; sh, short hairpin; NC, negative control; c-, cleaved.

Studies have found that the GSDM family induces cell death and inflammation; in particular, GSDMD is associated with pyroptosis (39-41). Activated caspase-1, -4, -5 and -11 cleave GSDMD protein into the GSDMD-N terminal with pore-forming activity (42). GSDMD-N combines with the cell inner membrane to form a pore, which causes cell swelling and osmotic lysis and induces pyroptosis (42). The present study showed that IRF2 silencing significantly suppressed pyroptosis-associated proteins, including GSDMD, GSDMD-N and c-caspase-1. The inflammatory response caused by MI is key to healing of heart trauma, however, it can also aggravate heart damage and dysfunction (12). MI activates the NLRP3 inflammasome, leading to the conversion of IL-1 $\beta$ and IL-18 into active mature forms in a caspase-1-dependent manner (43). Pro-inflammatory cytokines IL-1 and IL-18 expand infarct size and promote cardiac dysfunction (44). The present results showed that the expression levels of pro-inflammatory cytokines IL-1 $\beta$ and IL-18 increased in the MI group, and IRF2 silencing notably inhibited the expression levels of IL-1 $\beta$ and IL-18. Collectively, these data suggested that IRF2 silencing decreased myocardial injury by attenuating the pro-inflammatory response and decreasing pyroptosis.

Hypoxia stimulates myocardial cell damage and secretion of inflammatory factors, which can partially mimic heart damage in MI (26). Numerous studies have found that hypoxic stimulation activates the NLRP3 inflammasome and 
pyroptosis to induce cardiomyocyte death $(26,27)$. The present results showed that IRF2 silencing decreased the expression levels of IL-1 $\beta$, IL-18 and pyroptosis-associated proteins. H9c2 cells exposed to hypoxia were positively associated with IRF2 expression. The association between HIF1 and IRF2 was verified and it was shown that IRF2 expression may be regulated by the HIF-1 pathway. The present ChIP-PCR and luciferase reporter assays further demonstrated that IRF2 directly bound to the GSDMD promoter to drive GSDMD transcription for the execution of pyroptosis in hypoxia-stimulated H9c2 cells.

In conclusion, the present study demonstrated that IRF2 was key for MI and acts via increasing inflammasomes and pyroptosis. These findings confirmed that IRF2 was a key regulator of $\mathrm{MI}$ and provided novel insights into the mechanism of MI progression.

\section{Acknowledgements}

Not applicable.

\section{Funding}

No funding was received.

\section{Availability of data and materials}

The datasets used and analyzed during the current study are available from the corresponding author on reasonable request.

\section{Authors' contributions}

YL conceptualized and designed the study and wrote the manuscript. YL, YW and YH performed the experiments. HG and QW analyzed and interpreted the data. YL and YW confirm the authenticity of all the raw data. All authors read and approved the final version of the manuscript.

\section{Ethics approval and consent to participate}

The experimental protocol of the present study was performed in accordance with the Guide for the Care and Use of Laboratory Animals. All institutional and national guidelines for the care and use of laboratory animals were followed and the study was approved by the Ethics Committee of Cangzhou Central Hospital (approval no. CZSZ20200113).

\section{Patient consent for publication}

Not applicable.

\section{Competing interests}

The authors declare that they have no competing interests.

\section{References}

1. Benjamin EJ, Virani SS, Callaway CW, Chamberlain AM, Chang AR, Cheng S, Chiuve SE, Cushman M, Delling FN, Deo R, et al: Heart Disease and stroke statistics-2018 update: A report from the American heart association. Circulation 137: e67-e492, 2018.
2. Virani SS, Alonso A, Aparicio HJ, Benjamin EJ, Bittencourt MS, Callaway CW, Carson AP, Chamberlain AM, Cheng S, Delling FN, et al: Heart disease and stroke statistics-2021 update. Circulation 143: e254-e743, 2021.

3. Endorsed by the Latin American Society of Interventional Cardiology; PCI WRITING COMMITTEE, Levine GN, Bates ER, Blankenship JC, Bailey SR, Bittl JA, Cercek B, Chambers CE, Ellis SG, Guyton RA, et al: 2015 ACC/AHA/SCAI focused update on primary percutaneous coronary intervention for patients with ST-elevation myocardial Infarction: An update of the $2011 \mathrm{ACCF} / \mathrm{AHA} / \mathrm{SCAI}$ guideline for percutaneous coronary intervention and the 2013 ACCF/AHA guideline for the management of ST-elevation myocardial infarction: A report of the American College of Cardiology/American Heart Association Task Force on Clinical Practice Guidelines and the Society for Cardiovascular Angiography and Interventions. Catheter Cardiovasc Interv 87: 1001-1019, 2016.

4. Anderson JL, Adams CD, Antman EM, Bridges CR, Califf RM, Casey DE Jr, Chavey WE II, Fesmire FM, Hochman JS, Levin TN, et al: 2012 ACCF/AHA focused update incorporated into the ACCF/AHA 2007 guidelines for the management of patients with unstable angina/non-ST-elevation myocardial infarction: A report of the American College of Cardiology Foundation/American Heart Association Task Force on Practice Guidelines. J Am Coll Cardiol 61: e179-347, 2013.

5. Hausenloy DJ and Yellon DM: Myocardial ischemia-reperfusion injury: A neglected therapeutic target. J Clin Invest 123: 92-100, 2013.

6. van der Weg K, Prinzen FW and Gorgels AP: Editor's Choice-Reperfusion cardiac arrhythmias and their relation to reperfusion-induced cell death. Eur Heart J Acute Cardiovasc Care 8: 142-152, 2019.

7. Vanden Berghe T, Linkermann A, Jouan-Lanhouet S, Walczak H and Vandenabeele P: Regulated necrosis: The expanding network of non-apoptotic cell death pathways. Nat Rev Mol Cell Biol 15: 135-147, 2014.

8. Ji N, Qi Z, Wang Y, Yang X, Yan Z, Li M, Ge Q and Zhang J: Pyroptosis: A new regulating mechanism in cardiovascular disease. J Inflamm Res 14: 2647-2666, 2021.

9. Han Y, Qiu H, Pei X, Fan Y, Tian H and Geng J: Low-dose sinapic acid abates the pyroptosis of macrophages by downregulation of IncRNA-MALAT1 in rats with diabetic atherosclerosis. J Cardiovasc Pharmacol 71: 104-112, 2018.

10. Ibáñez B, Heusch G, Ovize M and Van de Werf F: Evolving therapies for myocardial ischemia/reperfusion injury. J Am Coll Cardiol 65: 1454-1471, 2015.

11. Toldo S and Abbate A: The NLRP3 inflammasome in acute myocardial infarction. Nat Rev Cardiol 15: 203-214, 2018.

12. van Hout GP, Bosch L, Ellenbroek GH, de Haan JJ, van Solinge WW, Cooper MA, Arslan F, de Jager SC, Robertson AA, Pasterkamp G and Hoefer IE: The selective NLRP3-inflammasome inhibitor MCC950 reduces infarct size and preserves cardiac function in a pig model of myocardial infarction. Eur Heart J 38: 828-836, 2017.

13. He WT, Wan H, Hu L, Chen P, Wang X, Huang Z, Yang ZH, Zhong CQ and Han J: Gasdermin D is an executor of pyroptosis and required for interleukin-1 $\beta$ secretion. Cell Res 25: 1285-1298, 2015.

14. Jia C, Chen H, Zhang J, Zhou K, Zhuge Y, Niu C, Qiu J, Rong X, Shi Z, Xiao J, et al: Role of pyroptosis in cardiovascular diseases. Int Immunopharmacol 67: 311-318, 2019.

15. Shi J, Zhao Y, Wang K, Shi X, Wang Y, Huang H, Zhuang Y, Cai T, Wang F and Shao F: Cleavage of GSDMD by inflammatory caspases determines pyroptotic cell death. Nature 526: 660-665, 2015.

16. Lei Q, Yi T and Chen C: NF- $\kappa$ B-Gasdermin D (GSDMD) axis couples oxidative stress and NACHT, LRR and PYD domains-containing protein 3 (NLRP3) inflammasome-mediated cardiomyocyte pyroptosis following myocardial infarction. Med Sci Monit 24: 6044-6052, 2018.

17. Han B, Xu J, Shi X, Zheng Z, Shi F, Jiang F and Han J: DL-3-n-butylphthalide attenuates myocardial hypertrophy by targeting gasdermin D and inhibiting gasdermin D mediated inflammation. Front Pharmacol 12: 688140, 2021.

18. Yanai H, Negishi $\mathrm{H}$ and Taniguchi T: The IRF family of transcription factors: Inception, impact and implications in oncogenesis. Oncoimmunology 1: 1376-1386, 2012.

19. Tamura T, Yanai H, Savitsky D and Taniguchi T: The IRF family transcription factors in immunity and oncogenesis. Annu Rev Immunol 26: 535-584, 2008. 
20. Heinz S, Haehnel V, Karaghiosoff M, Schwarzfischer L, Müller M, Krause SW and Rehli M: Species-specific regulation of Toll-like receptor 3 genes in men and mice. J Biol Chem 278: 21502-21509, 2003

21. Sun L, Jiang Z, Acosta-Rodriguez VA, Berger M, Du X, Choi JH, Wang J, Wang KW, Kilaru GK, Mohawk JA, et al: HCFC2 is needed for IRF1- and IRF2-dependent Tlr3 transcription and for survival during viral infections. J Exp Med 214: 3263-3277, 2017.

22. Kayagaki N, Lee BL, Stowe IB, Kornfeld OS, O'Rourke K, Mirrashidi KM, Haley B, Watanabe C, Roose-Girma M, Modrusan Z, et al: IRF2 transcriptionally induces GSDMD expression for pyroptosis. Sci Signal 12: eaax4917, 2019.

23. Warde-Farley D, Donaldson SL, Comes O, Zuberi K, Badrawi R, Chao P, Franz M, Grouios C, Kazi F, Lopes CT, et al: The GeneMANIA prediction server: Biological network integration for gene prioritization and predicting gene function. Nucleic Acids Res 38: W214-W220, 2010.

24. Bock-Marquette I, Saxena A, White MD, Dimaio JM and Srivastava D: Thymosin beta4 activates integrin-linked kinase and promotes cardiac cell migration, survival and cardiac repair. Nature 432: 466-472, 2004

25. Livak KJ and Schmittgen TD: Analysis of relative gene expression data using real-time quantitative PCR and the 2(-Delta Delta C(T)) Method. Methods 25: 402-408, 2001

26. Zhou Z, Wang Z, Guan Q, Qiu F, Li Y, Liu Z, Zhang H, Dong H and Zhang Z: PEDF inhibits the activation of NLRP3 inflammasome in hypoxia cardiomyocytes through PEDF receptor/phospholipase A2. Int J Mol Sci 17: 2064, 2016.

27. Chen A, Chen Z, Xia Y, Lu D, Yang X, Sun A, Zou Y, Qian J and Ge J: Liraglutide attenuates NLRP3 inflammasome-dependent pyroptosis via regulating SIRT1/NOX4/ROS pathway in H9c2 cells. Biochem Biophys Res Commun 499: 267-272, 2018.

28. Montojo J, Zuberi K, Rodriguez H, Bader GD and Morris Q: GeneMANIA: Fast gene network construction and function prediction for Cytoscape. F1000Res 3: 153, 2014.

29. Bellis A, Di Gioia G, Mauro C, Mancusi C, Barbato E, Izzo R, Trimarco B and Morisco C: Reducing cardiac injury during ST-elevation myocardial infarction: A reasoned approach to a multitarget therapeutic strategy. J Clin Med 10: 2968, 2021

30. Rymer JA, Fonseca E, Bhandary DD, Kumar D, Khan ND and Wang TY: Difference in medication adherence between patients prescribed a 30-day versus 90-day supply after acute myocardial infarction. J Am Heart Assoc 10: e016215, 2021.

31. England RN and Autieri MV: Anti-inflammatory effects of interleukin-19 in vascular disease. Int J Inflam 2012: 253583, 2012.

32. Cookson BT and Brennan MA: Pro-inflammatory programmed cell death. Trends Microbiol 9: 113-114, 2001.

33. Nazir S, Gadi I, Al-Dabet MM, Elwakiel A, Kohli S, Ghosh S, Manoharan J, Ranjan S, Bock F, Braun-Dullaeus RC, et al: Cytoprotective activated protein $\mathrm{C}$ averts Nlrp3 inflammasome-induced ischemia-reperfusion injury via mTORC1 inhibition. Blood 130: 2664-2677, 2017.
34. Dolunay A, Senol SP, Temiz-Resitoglu M, Guden DS, Sari AN, Sahan-Firat S and Tunctan B: Inhibition of NLRP3 inflammasome prevents LPS-induced inflammatory hyperalgesia in mice: Contribution of NF- $\kappa$ B, Caspase-1/11, ASC, NOX, and NOS Isoforms. Inflammation 40: 366-386, 2017.

35. Birnbaum MJ, van Zundert B, Vaughan PS, Whitmarsh AJ, van Wijnen AJ, Davis RJ, Stein GS and Stein JL: Phosphorylation of the oncogenic transcription factor interferon regulatory factor 2 (IRF2) in vitro and in vivo. J Cell Biochem 66: 175-183, 1997.

36. Li YR, Wen LQ, Wang Y, Zhou TC, Ma N, Hou ZH and Jiang ZP. MicroRNA-520c enhances cell proliferation, migration, and invasion by suppressing IRF2 in gastric cancer. FEBS Open Bio 6: 1257-1266, 2016.

37. Yongyu Z, Lewei Y, Jian L and Yuqin S: [ARTICLE WITHDR AWN] MicroRNA-18a Targets IRF2 and CBX7 to promote cell proliferation in hepatocellular carcinoma. Oncol Res 26: 1327-1334, 2018.

38. Liang C, Zhang X, Wang HM, Liu XM, Zhang XJ, Zheng B, Qian GR and Ma ZL: MicroRNA-18a-5p functions as an oncogene by directly targeting IRF2 in lung cancer. Cell Death Dis 8: e2764, 2017.

39. Shi J, Gao W and Shao F: Pyroptosis: Gasdermin-mediated programmed necrotic cell death. Trends Biochem Sci 42: 245-254, 2017.

40. Yuan B, Zhou XM, You ZQ, Xu WD, Fan JM, Chen SJ, Han YL, Wu Q and Zhang X: Inhibition of AIM2 inflammasome activation alleviates GSDMD-induced pyroptosis in early brain injury after subarachnoid haemorrhage. Cell Death Dis 11: 76, 2020.

41. Gao L, Dong X, Gong W, Huang W, Xue J, Zhu Q, Ma N, Chen W, Fu X, Gao X, et al: Acinar cell NLRP3 inflammasome and gasdermin D (GSDMD) activation mediates pyroptosis and systemic inflammation in acute pancreatitis. Br J Pharmacol 178: 3533-3552, 2021.

42. Liu X, Zhang Z, Ruan J, Pan Y, Magupalli VG, Wu H and Lieberman J: Inflammasome-activated gasdermin D causes pyroptosis by forming membrane pores. Nature 535: 153-158, 2016.

43. Deten A, Volz HC, Briest W and Zimmer HG: Cardiac cytokine expression is upregulated in the acute phase after myocardial infarction. Experimental studies in rats. Cardiovasc Res 55: 329-340, 2002.

44. Abbate A, Van Tassell BW, Seropian IM, Toldo S, Robati R, Varma A, Salloum FN, Smithson L and Dinarello CA: Interleukin-1beta modulation using a genetically engineered antibody prevents adverse cardiac remodelling following acute myocardial infarction in the mouse. Eur J Heart Fail 12: 319-322, 2010 .

(7) $\Theta$ This work is licensed under a Creative Commons Attribution-NonCommercial-NoDerivatives 4.0 International (CC BY-NC-ND 4.0) License. 